\section{$\underline{\text { Author Index }}$}

Abaigar, A

Abdalla, A L

Abdalla Filho, A L

Abdukader, A M

Abecia, L

Aberdi, O

Adam, $\mathrm{H}$

Adler, $\mathrm{S}$

Agarwal, N

Aguerre, M J

Aguilar, M

Alari, F O

Alazzeh, A Y

Alberdi, O

Alemu, A W

Alfaro, M A

Aljaloud, A A

Almeida, M T C

Almeida, R G

Almøy, T

Altermann, E

Altman, M B

Alves, B J R

Alves, T C

Amaral, G A

Amiro, B

Ammann, $\mathrm{C}$

Amon, B

Anais, C

Anderl, M

Anderson, D P

Anderson, $\mathrm{R}$

Angarita, E A

Angeles, M L

Annett, R

Anthony, $\mathrm{S}$

Antwi, C

Araujo, R C

Archimède, $\mathrm{H}$

Arco, A

Arif, A

Arndt, C

Arriaga, $\mathrm{H}$

Ascue, J

Astigarraga, L

Attwood, G T

Aubinet, $\mathrm{M}$

Aubry, A

Auldist, M J

Austermann, $\mathrm{F}$

Austin, K J

Auxiliadora Molina de

Almeida Teixeira, I

Avila-Stagno, $\mathrm{J}$

Azenha, M V

Azevedo, J A G

Bagnall, A

Bai, M

Baldock, J

Baldo, G L

Ball, B C

Balsari, $\mathrm{P}$

Bannink, A

Barbeiro, R

Barnett, M C
452,484

$336,346,347,381,382,383,385$

381,385

590

368,369

470

469

360

$414,425,479$

516,535

452,484

541

407

496

289

283,345

590

365

581

362

410

504

288

$501,519,521$

500, 537

289

284, 468

583

285

583

390

412

573,574

530

495

559

348

346,347

285,341

368

317

516,535

452, 470, 484, 496

314

$337,384,451$

406, 410

487, 489

321

405

467

372

523

486

540

578

445

$308,386,432$

327

527

281

266, 515

$272,363,499$

542

350
Barratt, C E

Barra, V

Barreda, D R

Baruah, L

Basarab, J A

Basurto Guriérrez, R

Batalla, M I

Battistotti, M

Baumgarten, A

Bayat, A R

Bayer, C

Beare, M H

Beauchemin, K A

Beautrais, J

Bébin, D

Beckers, Y

Becoña, G

Beegle, D

Behrendt, R

Bei, M

Beintmann, S

Belanche, A

Bell, M

Bell, M J

Bellocchi, G

Benoit, $\mathrm{M}$

Ben Touhami, $\mathrm{H}$

Berchielli, T T

Berger, C

Berndt, A

Berry, D

Berthiaume, R B

Bertilsson, J

Bertocco Ezequiel, J M

Besga, G

Beukers, A G

Bhatta, R

Biagini, D

Biechonski, M

Bishop-Hurley, G

Blasco, L

Boddey, R M

Bodson, B

Boehm, M T

Boland, T M

Bonnet, O J F

Boonyanuwat, $\mathrm{K}$

Boucher, D

Bouts, T

Boyd, G

Boyle, M

Bremner, G

Brennan, $P$

Briceño-Poot, E G

Bridget, B

Brito, A

Brito, L F

Bromley, A M

Browne, N A

Brunk, C

Buckley, F

Budan, A

Buendia Rodriguez, G

Bueno, A V I

Bull, I D

Bungenstab, D J

Burchill, W

Büscher, W
392

580

260, 326

307

388

530

450

584

583

274

505,537

426

$260,324,326,343,344,362,387$,

$388,389,510$

298

318

487,489

337,384

265

455

400

475

561,562

570

438,441

580

318

580

$408,409,449,456,503,538,540$

491

408, 409, 501, 519, 521

433

478

552

483

450

419

307

515

492

308,327

474

288

487

509

269, 292, 411, 416

505

587

262

317

527

484

440,579

334

338

395

518

503,540

352

455

345

279,433

577

530

448

555,563

287,581

506

$332,467,475$ 


\begin{tabular}{|c|c|}
\hline Byrne, K A & 453 \\
\hline Cabezas Garcia, E H & 588 \\
\hline Cabral Filho, S L S & 582 \\
\hline Cadenazzi, $\mathrm{M}$ & 384 \\
\hline Calif, B & 285 \\
\hline Calus, M P L & 462 \\
\hline Calvet, S & $444,471,496$ \\
\hline Cambareri, $\mathrm{S}$ & 461 \\
\hline Cambra-López, M & 471 \\
\hline Cameron, $\mathrm{K} \mathrm{C}$ & 281 \\
\hline Cammack, K M & 372 \\
\hline Campos, F C & 381 \\
\hline Campos, N M F & 500 \\
\hline Camp, V & 375 \\
\hline Cancer-Berroya, E & 313,320 \\
\hline Canibe, $\mathrm{N}$ & 589 \\
\hline Cantalapiedra-Hijar, G & 368 \\
\hline Canul-Solis, J R & 338 \\
\hline Capper, J L & $393,396,508$ \\
\hline Carberry, C A & 564 \\
\hline Cardenas, E A & 511,512 \\
\hline Cardenas, L & 465,559 \\
\hline Cardenas, L M & 313,320 \\
\hline Cardoso, A & 490,494 \\
\hline Cardoso, A S & 503,540 \\
\hline Carmona, G & 507 \\
\hline Carromeu, C & 581 \\
\hline Carson, A F & 278,356 \\
\hline Carulla, J E & 511,512 \\
\hline Carvalho, I P C & 408 \\
\hline Carvalho, M D & 525,538 \\
\hline Carvalho, P C F & $413,500,505,537$ \\
\hline Carvalho, W & 578 \\
\hline Casey, K D & 522 \\
\hline Casper, D P & 377 \\
\hline Castilho, L A & 382 \\
\hline Castilhos, Z M S & 500 \\
\hline Castro Lima, A R & 523 \\
\hline Castro Montoya, J M & 534,544 \\
\hline Cederberg, $\mathrm{C}$ & 442 \\
\hline Cerisuelo, A & 471 \\
\hline Cezimbra, I M & 505 \\
\hline Chadwick, D R & $283,313,320,375,559,560,566$ \\
\hline Chagunda, M G & 445 \\
\hline Chambers, B J & $375,560,566$ \\
\hline Chang, J & 464 \\
\hline Chang, J S & 428 \\
\hline Charbonneau, E C & 478 \\
\hline Charmley, E & $308,327,386$ \\
\hline Chatterton, $\mathrm{J} \mathrm{C}$ & 591 \\
\hline Chaturvedi, V B & 414 \\
\hline Chaudhary, L C & $414,425,479$ \\
\hline Chaves, A V & $407,419,422,486$ \\
\hline Chen, D & $308,400,472,524$ \\
\hline Chen, $\mathrm{X}$ & 543 \\
\hline Chesneau, G & 342 \\
\hline Choi, H B & 464 \\
\hline Choi, N J & 353,454 \\
\hline Chopin, $\mathrm{H}$ & 489 \\
\hline Christie, K M & 316 \\
\hline Christina Dias e Silva, N & 523 \\
\hline Chuko, B H & 363 \\
\hline Chung, Y H & 343 \\
\hline Cichota, $\mathrm{R}$ & 298 \\
\hline Cieslak, A & 492 \\
\hline Ciriaco, F M & 532 \\
\hline Clauss, M & 317 \\
\hline Clemens, G R & 426 \\
\hline Clough, T & 340 \\
\hline
\end{tabular}

\begin{tabular}{|c|c|}
\hline Clough, T J & 283 \\
\hline Cloy, $\mathrm{J}$ & 465 \\
\hline Coates, T W & 308 \\
\hline Cocks, B G & 312 \\
\hline Coleman, M D & 480 \\
\hline Cole, N A & 469,504 \\
\hline Collins, R P & 361 \\
\hline Conant, G C & 372 \\
\hline Cone, J W & 363 \\
\hline Consortium, Feed Efficiency & 397 \\
\hline Cookson, A L & 406 \\
\hline Cordovín, L & 452,484 \\
\hline Correa Santos, V & 483 \\
\hline Corson, M S & 342 \\
\hline Corton, $\mathrm{J}$ & 459 \\
\hline Costa, C C M & 525,538 \\
\hline Coutinho, $\mathrm{J}$ & 497 \\
\hline Cowan, $\mathrm{N}$ & 570 \\
\hline Cox, F & 404 \\
\hline Craigon, J & 438,441 \\
\hline Creevey, C J & 564 \\
\hline Crompton, L A & $364,391,392$ \\
\hline Crosson, $\mathrm{P}$ & 257,334 \\
\hline Cross, $\mathrm{P}$ & 322 \\
\hline Cuk, D & 266 \\
\hline Cullen, B R & 401,403 \\
\hline Curwen, A & 591 \\
\hline Daatselaar, C H G & 373 \\
\hline Dalantonia, E & 449,456 \\
\hline Danielsson, $\mathrm{R}$ & 552 \\
\hline Dansen, $\mathrm{O}$ & 502,517 \\
\hline Dardenne, $\mathrm{P}$ & 279,433 \\
\hline Da Silva, C S & 505 \\
\hline Datta, A & 465 \\
\hline D'Aurea, A P & 302,365 \\
\hline Davies, J & 359 \\
\hline Davison, T B & 567 \\
\hline Debacq, A & 489 \\
\hline De Blas, C & 471 \\
\hline De Boer, I J M & 263,300 \\
\hline De Campeneere, S & $367,534,544$ \\
\hline De David, D B & 537 \\
\hline Deerhake, M E & 267 \\
\hline De Haas, Y & $378,447,462$ \\
\hline Dehareng, F & 279,433 \\
\hline Deighton, M H & $\begin{array}{l}269,279,405,411,416,421,423, \\
433\end{array}$ \\
\hline de Klein, C A M & 283,304 \\
\hline Delaby, L & 290,342 \\
\hline de la Fuente, G & 561,562 \\
\hline Delevatti, L M & $409,449,456$ \\
\hline del Hierro, O & 450 \\
\hline Dell, C J & 265 \\
\hline Demande, $\mathrm{N}$ & 539 \\
\hline Demeyer, P & 482 \\
\hline De Neve, J & 526 \\
\hline Denman, S E & $259,349,430$ \\
\hline Denmead, O T & 400,524 \\
\hline Denonfoux, J & 262 \\
\hline de Oliveira, E M & 483 \\
\hline Derno, $\mathrm{M}$ & 374,513 \\
\hline Dersch, G & 583 \\
\hline Desjardins, R L & 380,472 \\
\hline Deswysen, D & 539 \\
\hline De Vries, J W & 263,477 \\
\hline Dewhurst, R J & $325,555,563$ \\
\hline Dewulf, J & 439 \\
\hline Dhali, A & 307 \\
\hline Di, H J & 281 \\
\hline
\end{tabular}




\begin{tabular}{|c|c|}
\hline Dijkstra, J & $\begin{array}{l}272,363,378,434,451,499,502, \\
517\end{array}$ \\
\hline DiLorenzo, $\mathrm{N}$ & 532 \\
\hline Ding, $\mathrm{S}$ & 407 \\
\hline Dini, Y & 451 \\
\hline Dinuccio, E & 266,515 \\
\hline Dittert, K & 524 \\
\hline Dittmann, M T & 317 \\
\hline Dludla, S P & 294,295 \\
\hline Dodds, K G & 412 \\
\hline Döhler, H & 528 \\
\hline Dolle, J B & 457 \\
\hline Dolman, M A & 373 \\
\hline Dong, L F & 278 \\
\hline Donnellan, $\mathrm{T}$ & 257 \\
\hline Donovan, $\mathrm{N}$ & $313,320,375,392$ \\
\hline Doran, $\mathrm{S}$ & 459,460 \\
\hline Doreau, $\mathrm{M}$ & $275,285,341,342$ \\
\hline Dorfner, G & 493 \\
\hline Drury, C & 461 \\
\hline Dugat-Bony, E & 262 \\
\hline Dumortier, $\mathrm{P}$ & 489 \\
\hline Dunfield, K & 319 \\
\hline Dunlop, C J & 426 \\
\hline Dupard, P & 484 \\
\hline Duthie, C A & $376,545,548,551,569,575$ \\
\hline Du Toit, C J L & 558 \\
\hline du Toit, P C V & 431 \\
\hline Duval, B D & 264 \\
\hline Duval, S M & $260,261,326,368,387,389$ \\
\hline Dyer, J A & 380 \\
\hline Eckard, R J & $\begin{array}{l}269,282,316,401,403,411,416, \\
424,441,455\end{array}$ \\
\hline Edwards, G R & 325 \\
\hline Elhottova, D & 427 \\
\hline Ellis, J L & 499 \\
\hline Ellison, M J & 372 \\
\hline El-Zaiat, H M & 346,347 \\
\hline Emmenegger, L & 429,554 \\
\hline Emmerling, $\mathrm{M}$ & $303,305,402$ \\
\hline Engberg, R M & 589 \\
\hline Escobar, $\mathrm{P}$ & 324 \\
\hline Estellés, F & $444,471,496$ \\
\hline Eugène, $\mathrm{M}$ & $275,285,342,531,533$ \\
\hline Evans, L & 268 \\
\hline Evans, M J & 352 \\
\hline Everts, $\mathrm{H}$ & 517 \\
\hline Ezequiel, J M B & 302,365 \\
\hline Faborode, $\mathrm{M} \mathrm{O}$ & 399 \\
\hline Fadel, J G & 377 \\
\hline Fadul-Pacheco, L F P & 478 \\
\hline Fagundes, G M & 385 \\
\hline Falchero, L & 526 \\
\hline Famulari, D & 570 \\
\hline Fangueiro, D & 497 \\
\hline Farrelly, N & 257 \\
\hline Faulkener, C & 473 \\
\hline Favaro, V R & 365 \\
\hline Fávaro, V R & 302 \\
\hline Felber, R & 468 \\
\hline Feldkamp, C R & 287 \\
\hline Fernandes, E H & 302 \\
\hline Fernández, C & 444 \\
\hline Ferreira, E J & 339 \\
\hline Ferrer, $\mathrm{P}$ & 471 \\
\hline Ferris, C P & 278,356 \\
\hline Feyereisen, G W & 265 \\
\hline Fievez, V & $367,526,544,577$ \\
\hline Finnan, $\mathrm{J}$ & 257 \\
\hline
\end{tabular}

Fitton, N 465

Fleming, $\mathrm{H} \quad 358,359,361$

Flesch, T K 308, 469, 472

Fock, T 287

Fon, F N 294, 295

Fonseca, E L 315, 498

Fontana, D C 413

Fragoso, R 484

Fraisse, C W 413, 529

Francisco Ventoso

Bompadre, $\mathrm{T} \quad 523$

Franco, F E 273, 584

Franco, J 337

Franger, L 589

Frankel, T 303, 305, 402

Franks, A 303, 305, 402

Fraser, M 459

Fraser, M D 358, 359, 361

Friedman, N 571

Friggens, N C 300

Friguetto, R 542

Friguetto, R T S 408, 409, 541

Froidmont, E 279, 433

Fuchs, C 287

Fuhrer, J 284

Fuller, R 576

Gac, A 457

Gallard, Y 342

García-Rebollar, P $\quad 471$

Gardiner, T D 480

Garnett, E J 436

Garnsworthy, P C 438, 441

Gemeda, B S 485

Gengler, N 279, 433, 488

Gere, J I 537

Ghini, R 385

Gibbs, J 340

Gidlund, $\mathrm{H} \quad 588$

Gilhespy, S 559

Gioelli, F 266

Giraldo, L A $\quad 514,520$

Giraldo, P 514, 520

Godwin, I R 350

Godwin, S 277

Goel, N 329

Gomez Rosales, S 530

Gonçalves, L C G 578

Gonda, H 552

Gonda, H L 537

Gooch, C 568

Goodman, J R 438

Gordon, R J 268, 370, 461, 466

Grace, P 283, 345

Grant, R H 509

Graviou, D 262

Green, C 364, 391

Griffith, D W T 400, 524

Griggs, T C 323

Groot Koerkamp, P W G 378

Grover, S 340

Grover, S P P 357

Guan, L 260, 326

Guan, L L 387

Guerouali, A 286, 536

Guilet, D 577

Guimarães Junior, R $\quad 582$

Gulino, L M 277

Gutierrez-Zamora J M L 277

Guyader, J 275 
Guzman, C

Haeussermann, U

Haisan, J

Hammami, $\mathrm{H}$

Hammond, K J

Hammon, $\mathrm{H}$

Hannah, M C

Hanrahan, K

Harland, R

Harper, L A

Harrison, J

Harrison, M T

Harstad, O M

Hart, K J

Harvey, M J

Hasenauer, $\mathrm{H}$

Hassen, A

Hateley, G

Hatew, B

Hatton, V

Hawkins, G

Hayes, B J

Hayes, D J

Hebel, C

Hegarty, R S

Heinesch, B

Heißenhuber, A

$\mathrm{He}, \mathrm{J} Z \mathrm{Z}$

Helena Machado Rocha F M 523

Hellwing, A L F 556, 557

He, M L

Henderson, G

Hendren, Z D

Hendriks, J

Hendriks, W H

Henriksson, $\mathrm{M}$

Henry, D D

Herman, L

Hessle, A

Hetta, M

Hickey, S H

Hill, J

Hindrichsen, I K

Hippenstiel, F

Hodgson, C J

Hoffmann, $\mathrm{H}$

Hofmann, G

Højberg, O

Hollier, J

Holo, $\mathrm{H}$

Hölscher, R

Homem Júnior, A C

Hoogeveen, M W

Horan, B

Houdijk, J

Hou, F

Hristov, A N

Huang, X D

Hube, $S$

Huhtanen, P

Humphreys, J

Humphries, D J

Hunter, M

Hutchings, N J

Huysveld, S

Hyndman, D

Hyslop, J J

Issakowicz, J

Jacobs, J L
$303,305,402$

528

260,326

488

364,391

374

405, 421

257

412

469

568

316

$343,360,362,407,486$

473

283,352

583

431,485

591

272,499

587

568

312

508

317

$350,440,446,553,579$

489

493

281

407

404, 406

267

435

502,517

333,442

532

482

333

588

412

400,567

434

475

313,320

493

493

589

282

407

467

$302,365,483$

373

481

548

543

265

430

345

518,588

506

261, 364, 391

438

291

439

412

$376,545,548,551,569,575,576$

382,383

328,423
Jacobs, M P T

Jacobson, L

Janssen, P H

Jantalia, C P

Janusckiewicz, E R

Janzen, H H

Jayanegara, A

Jayasundara, S

Jayme, D G

Jensen, B B

Jérôme, E

Jeyanathan, J

Jiao, H P

Jirout, J

Jocher, M

Johannesson, G

Johnson, K A

Johnston, C J

Jo, N C

Jones, A K

Jones, D L

Jørgensen, M S

Junqueira, J

Kage, $\mathrm{H}$

Kahala, M

Kairenius, P

Kamra, D N

Kandel, P B

Kang, A

Kantelhardt, J

Kanu, A U

Kaspar, M

Kaye, J

Kebreab, E

Keck, M

Kelliher, F

Kelliher, K M

Kelly, K B

Kelly, W J

Kenny, D A

Kentler, C

Kienzle, M

Kilic, U

Kim, C H

Kim, E J

Kim, S H

Kim, S S

Kim, Y S

Kindermann, $\mathrm{M}$

Kingwell, R S

Kinh, L V

Kinley, R D

Kirton, $\mathrm{P}$

Kittelmann, S

Kitzler, B

Kjestrup, $\mathrm{H}$

Klieve, A V

Klop, G

Klumpp, K

Knox, P

Kohmann, M M

Kolte, A

Köster, J R

Koury Filho, W

Kreuzer, M

Kristensen, T

Krizsan, S J

Kuhla, B

Kuikman, P J
502,517

568

404

288

540

510

293

461

578

589

487, 489

565

356

427

284

268

$393,396,518,536$

509

454

322

322

291

490,494

524

474

274

$414,425,479$

$279,433,488$

277

583

301

583

265

$289,348,377,451$

554

340

283

282,328

406, 410

564

303,305

277

473

353

428, 464

428,582

464

464

261, 368

455

587

272

261, 364, 391

404

583

273, 412, 584

277

272

431,580

568

529, 532, 537

307

524

541

317,458

333

588

374,513

366 


\begin{tabular}{|c|c|c|c|}
\hline Kume, S & 306 & Lucas, R C & 336 \\
\hline Kupper, T & 549 & Ludemann, C I & 424 \\
\hline Kurekci, C & 259 & Luijben, $\mathrm{K}$ & 440,579 \\
\hline Kushwaha, B P & 547 & Lund, $\mathrm{P}$ & $556,557,589$ \\
\hline $\mathrm{Ku}-$ Vera, J C & 338 & Luo, D W & 271 \\
\hline Laabouri, F Z & 286 & Luo, J & 395 \\
\hline Lage, J F & $408,409,449,456,542$ & MacAdam, J W & 323 \\
\hline Laidlaw, A S & 550 & Macedo, S & 497 \\
\hline Laker, M C & 435 & Machado, F S & 578 \\
\hline Lalor, S & 257,484 & Machado, M & 456 \\
\hline Lalor, S T J & 453 & MacLean, S & $273,412,584$ \\
\hline Lamberson, W R & 372 & Madalena, G & 540 \\
\hline Lamb, G C & 532 & Madari, B E & 288 \\
\hline Lambie, S C & 406,410 & Madsen, J & 556,557 \\
\hline Lanigan, G J & $257,453,484,506$ & Magalhaes, F A & 578 \\
\hline Lardy, R & 580 & Mahabalarao, M L & 331 \\
\hline La Scala, N & 540 & Maia, A S C & 525,538 \\
\hline Lassen, J & 280 & Maldaner, L & 268 \\
\hline Lassey, K R & 269,416 & Malheiros, E B & 541 \\
\hline Laubach, J & $273,340,352,357$ & Mali, D J & 572 \\
\hline Lauwers, L & 439 & Mamuad, L & 428 \\
\hline Lauzon, J & 461 & Manefield, M & 277 \\
\hline Lazzaroni, C & 515 & Manneville, $\mathrm{V}$ & 457 \\
\hline Leahy, S C & 406,410 & Mäntysaari, E A & 463 \\
\hline Ledgard, S & 395 & Mäntysaari, P & 463 \\
\hline Lee, $\mathrm{C}$ & 265 & Marett, L & 312 \\
\hline Leemans, D & 459,460 & Marie-Magdeleine, $\mathrm{C}$ & 285,341 \\
\hline Lee, S S & 428,464 & Marin, A & 514,520 \\
\hline Lee, S Y & 454 & Marques, R & 501 \\
\hline Leifeld, J & 284 & Martí, J V & 444 \\
\hline Leip, A & 476,507 & Martin, C & $275,285,491,565$ \\
\hline Lemos, N L S & 541 & Martinez-Fernandez, G & 368,369 \\
\hline Leskinen, H M & 274 & Martin-Garcia, A I & 368,369 \\
\hline Lessa, A C de R & 288 & Martini, S & 323 \\
\hline Lesschen, J P & $258,366,476$ & Martin-Neto, L & 339 \\
\hline Lettat, A & 491 & Martin, R J & 352 \\
\hline Levy, P & 570 & Mathot, M & 539 \\
\hline Lewis, E & 279,433 & Matlock, M & 527 \\
\hline Lewis, $\mathrm{K}$ & 369 & Matsui, $\mathrm{H}$ & 349 \\
\hline Lherm, M & 318 & Maxime, D & 380 \\
\hline $\mathrm{Li}, \mathrm{B}$ & 296 & Mayorga, O L & $511,512,573,574$ \\
\hline $\mathrm{Li}, \mathrm{C}$ & 559,585 & Mbiriri, D T & 353 \\
\hline $\mathrm{Li}, \mathrm{D}$ & 410,506 & McAllister, T A & $343,344,362,407,486,510$ \\
\hline Lidauer, M H & 463 & McBride, $\mathrm{J}$ & 299 \\
\hline Liinamo, A E & 463 & McCartney, C A & 555,563 \\
\hline $\mathrm{Li}, \mathrm{L}$ & 440,579 & McConkey, B & 380 \\
\hline Lindahl, C & 333 & McDowell, D A & 278,356 \\
\hline Lindsey, $\mathrm{S}$ & 395 & McEwan, J C & 412,584 \\
\hline Little, S M & 388,510 & McEwan, N R & 270 \\
\hline Liu, J & 259 & McFarlane, J R & 350 \\
\hline Liu, J X & 355,398 & McGeough, E J & $343,344,510$ \\
\hline Liu, M & 398 & McGinn, S M & $308,343,344,387,400,472,510$ \\
\hline Liu, Y & 543 & McGrath, J & 440 \\
\hline Lively, F & 550 & McManus, C & 382 \\
\hline $\mathrm{Li}, \mathrm{Y}$ & 309,410 & McMillan, A M S & 352 \\
\hline Lizarralde, $\mathrm{C}$ & 384 & McParland, S & 279,433 \\
\hline Lohoelter, M & 513 & McPhee, M J & 446 \\
\hline Loh, Z & 400 & McSweeney, C & 430 \\
\hline Loncke, $\mathrm{C}$ & 531 & McSweeney, C S & 259,349 \\
\hline Long, $\mathrm{R}$ & 430 & Meale, S J & $407,419,422,486$ \\
\hline López, D M & 470 & Medina, B & 367 \\
\hline López, M C & 444 & Meenken, E D & 426 \\
\hline Lorinquer, E & 457 & Meile, L & 458 \\
\hline Losa, R & 367 & Meissner, H H & 558 \\
\hline Lourenço, M & 526 & Meister, N C & 541 \\
\hline \multirow[t]{2}{*}{ Louvandini, $\mathrm{H}$} & $336,346,347,381,382,383,385$ & Mendez, J J & 530 \\
\hline & & Menegueli, F & 542 \\
\hline Løvendahl, P & 280 & Menzi, $\mathrm{H}$ & 549 \\
\hline Loyon, L & 484 & Mercadante, V R G & 532 \\
\hline
\end{tabular}


Merino, $\mathrm{P}$

Metges, C

Meyer, U

Michal, J

Michal, J J

Milinovich, G

Miller, J

Milori, D M B P
Miri, V H

Mishra, A K

Misselbrook, T H

Mitchell, R A

Mitsumori, M

Mizrahi, I

Moate, P J

Modernel, $\mathrm{P}$

Moeletsi, M E

Mogensen, L

Mohini, M

Mohn, J

Mokolobate, M C

Molano, G

Molina-Alcaide, E

Moniuszko-Szajwaj, B

Montoneri, E

Moorby, J M

Moraes, A B

Moraes, L E

Moraes, $\mathrm{P}$

Moran, D

Moreau, S

Morgado, E S

Morgavi, D P

Morrison, S J

Morsy, A S

Moser, T

Moset, V

Moureaux, C

Muetzel, S

Mühling, K H

Muir, S K

Mukherjee, S

Mukhtar, S

Murray, R

Murray, R L

Muylle, $\mathrm{H}$

Nag, S K

Nan, Z

Nape, M K

Nascimento, S T

Natel, A S

Naylor, T

Neftel, A

Negussie, E

Neser, F W C

Neto, A J

Newbold, C J

Nguyen, G

Nguyen, $\mathrm{H}$

Nguyen, T T H

Ngwabie, N M

Nielsen, N I

Nishida, $\mathrm{T}$

Noel, S

Nogueira, A R A

Nolan, J V
444, 452, 470, 484, 496

374

513

518

536

589

440,553

339

404

547

$313,320,375,392,560,566$

401,403

349

571

$269,312,405,411,416,421,422$, 423,441

384

379

333

$329,330,331$

429

435

$271,273,357,412,584$

368

492

515

$358,359,361,459,460,561$

500

377

527

586

457

503,540

$262,275,285,341,367,485,491$, 533,565

299,356

$336,346,347$

583

471

487

335,420

524

328,400

352

390,568

313

320

439

547

543

379

525,538

385

$400,432,446$

284

463,474

435

449

$270,335,367,369,437,473,561$, 562

533

533

342

268

333

394

404

519,521

350
Nooriyan Soroor, E $\quad 371$

Norberto, N M 578

Nordberg, $\AA \quad 314$

Norris, S 313, 320

Novinski, C O 448

Nowak, A 492

Nozière, $P \quad 275,491$

Nsahlai, I V 294, 295

Nuñez, C 367

Nwogbugbo, I 301

O'Brien, M 394

O'Connell, N E 495

Oba, M

O'Brien, D

O'Brien, M

$260,324,326,387$

$257,481,493$

292

301

440

366

378,447

399

292, 334

387,389

546

542

302

$339,501,519,521$

503

289

513

339

277

511,512

$271,304,395,420$

524

259,430

484

382,383

288

262

454

464

365

501

483

346,347

417,418

494

415

361

479

382

492

$501,519,521$

519,521

317

$439,534,544$

478

$363,499,502,517$

498

553,579

335,440

518

578,582

285

406

492

262

262

$339,501,519,521$ 


\begin{tabular}{|c|c|c|c|}
\hline Pfeifer, F M & 505 & Ross, D W & $376,545,551,569,575$ \\
\hline Phelps, D & 403 & Ross, E M & 312 \\
\hline Phillips, F A & $400,432,446$ & Rosseto, E & 503 \\
\hline Phuong, H N & 300 & Rossi Junior, P & 448 \\
\hline Picasso, V & 337,384 & Roth, U & 528 \\
\hline Picasso, V D & 451 & Rotz, C A & 276 \\
\hline Pickering, N K & 412 & Roulenc, M & 318 \\
\hline Pierre, C & 577 & Rouzbehan, Y & 371 \\
\hline Pierre, D & 487 & Rowell, D & 400 \\
\hline Pinares-Patiño, C S & $273,357,412,436,584$ & Rowlings, D & 345 \\
\hline Pineau, J & 531 & Ruelle, E & 290 \\
\hline Pineras, $\mathrm{C}$ & 518 & Ruggieri, A C & $490,494,503,540,541$ \\
\hline Pinto, $\mathrm{M}$ & 450 & Ruiz Favaro, V & 483 \\
\hline Pinto, $\mathrm{S}$ & 448 & Ruiz-Moreno, M & 532 \\
\hline Pires, A V & 347 & Ryu, C H & 353 \\
\hline Podesta, S C & $272,363,499$ & Saetnan, E R & 437 \\
\hline Podolyan, A & 281 & Sagoo, L & 560,566 \\
\hline Poli, C H E C & 500 & Sakthivel, P C & 414 \\
\hline Popova, M & 262,341 & Salas, W & 559,585 \\
\hline Popp, J & 585 & Sallam, S M A H & 336 \\
\hline Possas, F P & 578 & Salomon, E & 354 \\
\hline Potterton, S L & 438 & Samal, L & 425 \\
\hline Pötzelsberger, E & 583 & Sampaio, A C K & 382,383 \\
\hline Poulsen, M & 589 & Sanderson, R & 358 \\
\hline Powell, J M & $264,276,516,535$ & Sandoval, E & $273,412,584$ \\
\hline Powers, C & 568 & Sanni, L A & 399 \\
\hline Prasad, C S & 307 & Santana, M M & 505 \\
\hline Pries, M & 475 & Santana, S S & 540 \\
\hline Pronto, J & 568 & Santos, J E & 383 \\
\hline Prosenbauer, M & 583 & Santos, S & 542 \\
\hline Pszczola, M & 462 & Santos, V C & 302,365 \\
\hline Pulleman, M M & 477 & San Vito, E & $408,409,449,456$ \\
\hline Ramírez-Avilés, L & 338 & Saravanan, M & 307 \\
\hline Ramirez-restrepo, C & 327 & Saunders, N & 438 \\
\hline Rawnsley, R P & 316 & Savian, J V & $500,505,537$ \\
\hline Rees, B & $465,559,570$ & Schaller, L & 583 \\
\hline Reijs, J W & 373 & Scheer, C & 345 \\
\hline Reis, L G & 422 & Schiefler, I & 332,475 \\
\hline Reis, R A & $408,503,540,542$ & Schleper, C & 589 \\
\hline Rendon, J A & 348 & Schmidely, P H & 300 \\
\hline Reynolds, C K & $261,364,391,392$ & Schmidt, D & 568 \\
\hline Ribas, M N & 578 & Schmidt, $\mathrm{P}$ & 448 \\
\hline Ribaux, B E & 422 & Schmithausen, A & 332 \\
\hline Ribeiro, A F & $408,409,449,456$ & Scholtz, M M & 435 \\
\hline Ribeiro Junior, G O & $407,486,578$ & Schrade, S & 429,554 \\
\hline Ricci, P & 548 & Schramm, A & 589 \\
\hline Richmond, A S & 550 & Schulte, R & 257 \\
\hline Rira, M & 285,341 & Schwab, C & 589 \\
\hline Risse, M & 568 & Schwarm, A & 513 \\
\hline Rivera Rivera, A & 523 & Schweigel-Roentgen, M & 513 \\
\hline Rizopoulus, A & 402 & Scogings, P F & 294,295 \\
\hline Roberts, D G & 445 & Segnini, A & 339 \\
\hline Robinson, D L & 440,553 & Seo, K S & 454 \\
\hline Rochette, $\mathrm{P}$ & 283 & Seo, $\mathrm{S}$ & 353 \\
\hline Rochette, Y & 262,491 & Seo, S W & 454 \\
\hline Rochford, N M & 453 & Serra, J & 542 \\
\hline Rodhe, L K K & 314,354 & Shalloo, L & $257,290,481,493$ \\
\hline Rodríguez, C & 471 & Shearer, A & 367 \\
\hline Rodriguez, H G & 585 & Sherlock, R & 340 \\
\hline Roehe, $\mathrm{R}$ & $376,545,548,551,569$ & Shingfield, K J & 274,474 \\
\hline Roessler, R & 528 & Shinkai, T & 349 \\
\hline Rolle, L & 266 & Shoda, M & 394 \\
\hline Rollo, M D & 304 & Sigmund, E & 583 \\
\hline Romanzini, E & 542 & Silberberg, M & 491 \\
\hline Romero Perez, A & 387,389 & Silou-Etienne, $\mathrm{T}$ & 341 \\
\hline Röntgen, M & 374 & Silva, L R F & 336 \\
\hline Rood, T & 258 & Silva, R A & $408,409,456$ \\
\hline Rooke, J A & $376,445,545,551,569,575$ & Silva, R B & 525,538 \\
\hline Rosato, R & 515 & Silva, V C & 541 \\
\hline
\end{tabular}




\begin{tabular}{|c|c|}
\hline Simek, M & 427 \\
\hline Simonetti, L & 456 \\
\hline Singh, A & 547 \\
\hline Singh, B & 331 \\
\hline Singh, $\mathrm{S}$ & 547 \\
\hline Sirohi, S & $329,330,331$ \\
\hline Sithambaram, S & 587 \\
\hline Skiba, U & 570 \\
\hline Smith, A H & 344 \\
\hline Smith, D W & 390,568 \\
\hline Smith, E L & 370 \\
\hline Smith, K & 465 \\
\hline Smith, K F & 424 \\
\hline Smith, P & 465,559 \\
\hline Snider, D & 319,461 \\
\hline Soltan, Y A & $336,346,347$ \\
\hline Song, J Y & 464 \\
\hline Soriano, A & 428 \\
\hline Soussana, J F & 431 \\
\hline Souza, C E & 582 \\
\hline Souza, C M & 448 \\
\hline Soyeurt, H & $279,433,488$ \\
\hline Spang, A & 589 \\
\hline Sperança, M A & 339 \\
\hline Spleth, P & 333 \\
\hline Stefański, T & 274 \\
\hline Stefenson Ribeiro Junior, C & 523 \\
\hline Steinberg, W & 261 \\
\hline Stilmant, D & 539 \\
\hline Storlien, T M & 360,362 \\
\hline Stowell, R & 568 \\
\hline Strabel, T & 462 \\
\hline Strathe, A & 377 \\
\hline Sudarman, A & 293 \\
\hline Südekum, K H & 475 \\
\hline Sulaiman, F & 461 \\
\hline Sundberg, M & 354 \\
\hline Sun, $\mathrm{X} Z$ & 271,395 \\
\hline Sun, Y & 260,326 \\
\hline Sun, Z Y & 355 \\
\hline Svendsen, G T & 546 \\
\hline Swain, H M & 529 \\
\hline Swensson, C & 333,442 \\
\hline Szczechowiak, J & 492 \\
\hline Szumacher-Strabel, M & 492 \\
\hline Szymczak, M & 492 \\
\hline Taghizadeh-Toosi, A & 340 \\
\hline Tajima, K & 349 \\
\hline Takahashi, J & 293,394 \\
\hline Takenaka, A & 349 \\
\hline Taki, R & 461 \\
\hline Talore, D G & 431 \\
\hline Tapio, I & 274,474 \\
\hline Tava, A & 526 \\
\hline Tavendale, $\mathrm{M}$ & 420 \\
\hline Taylor, U & 576 \\
\hline Teixeira, A M & 578 \\
\hline Tenuta, $\mathrm{M}$ & 289 \\
\hline Teresinha, T & 542 \\
\hline Tesfamaraim, E & 431 \\
\hline Tessier, $\mathrm{N}$ & 367,577 \\
\hline Thas, O & 526 \\
\hline Theobald, V J & 358 \\
\hline Thirlwell, D & 576 \\
\hline Thoma, G & 527,585 \\
\hline Thomas, S M & 426 \\
\hline Thompson, $\mathrm{K}$ & 319 \\
\hline Thorman, R E & $375,465,560,566$ \\
\hline Thorne, F & 257 \\
\hline
\end{tabular}

Thuen, E 360

Tilston, E 559

Timms, M 403

Tischler, M R 505

Todd, R W 469, 504

Tomás Resende, K 523

Tomich, T R 578

Tomkins, N 308, 386, 432

Topp, K 465, 559

Torre Neto, A 385

Toyoko Shiraishi Frighetto, R 523

Trentin, C B 315

Trindade, $\mathrm{H} \quad 497$

Troy, S 551

Turner, D A 400

Ubaka-Blackmoore, N C 267

Ukachukwu, S N $\quad 301$

Ulrich, R 585

Unamunzaga, O 450

Urich, T 589

Urquiaga, S 288

Utsumi, S 518

Vadas, P A 264

Vaddella, V K 522

Valdivia-Salgado, V 338

Vale, J 459

Valente, A 542

Vallente, A L S 540

Vandaele, L 534

Van Den Berg, W 477

van der Werf, H M G 342

VanderZaag, A C 268, 370, 380, 461

Van Doorn, D A 502,517

Vandré, R 528

Van Eenennaam, A L $\quad 348$

Van Heyst, W J 572

Van Laar, H 272, 499

Vanlierde, A 279, 433

Van linden, V 439, 482

van Niekerk, W A 558

Van Riel, J W 447

Vanrobays, M L 279, 488

Van Rooyen, L 563

Vargas, J J 511, 512

Vasconcelos, E 497

Veerkamp, R F 447, 462

Vejlin, J 291

Velasco, F O 578

Velazco, J I 579

Veneman, J B 335, 437

Venterea, R T 283

Ventto, L 274, 474

Vercoe, $\mathrm{P} \quad 432$

Vergé, X P C 380

Vermaut, $\mathrm{S} \quad 539$

Vestergaard, M 333

Veysset, P 318

Vieira, B R 449

Viguria, M 470

Vilkki, J 274, 474

Vogeler, I 298

Volden, H 362

Vollmer, M K 429

Vyas, D 344

Waddams, K E 270

Waghorn, G C 404, 436, 518

Wagner, A P L 413

Wagner-Riddle, C 268, 319, 370, 461, 466, 572

Wagner, $\mathrm{S} \quad 258$ 


\begin{tabular}{|c|c|}
\hline Wales, W J & 423 \\
\hline Wallace, M & 290 \\
\hline Wallace, R J & $376,545,569$ \\
\hline Wall, E & 462 \\
\hline Wang, J K & 355,398 \\
\hline Wang, L Z & $309,310,311$ \\
\hline Wang, M & 296,297 \\
\hline Ward, G N & 282,328 \\
\hline Wareing, S & 576 \\
\hline Waterhouse, A & $376,548,569,575$ \\
\hline Waterhouse, $\mathrm{T}$ & 545,551 \\
\hline Waters, S M & 564 \\
\hline Watkins, N & 395 \\
\hline Wattiaux, M A & $276,516,535$ \\
\hline Weber, K L & 348 \\
\hline Weckwerth, W & 589 \\
\hline Weisbjerg, M R & 556,557 \\
\hline Weiss, F & 476,507 \\
\hline Westhoek, H J & 258,476 \\
\hline Wheadon, N M & 325 \\
\hline Whip, P & 403 \\
\hline Whitefield, E & 568 \\
\hline White, R R & $393,396,536$ \\
\hline Widiawati, Y & 443,587 \\
\hline Wilcox, $\mathrm{R}$ & 438 \\
\hline Wilfart, A & 533 \\
\hline Willén, A & 314 \\
\hline Williams, A G & 591 \\
\hline Williams, J R & $375,465,560,566$ \\
\hline Williams, M & 506 \\
\hline Williams, S R O & $269,405,411,416,421,422,423$ \\
\hline Wilson, J D & 469,472 \\
\hline Wina, E & 293 \\
\hline Winiwarter, W & 583 \\
\hline Wood, J D & 370,466 \\
\hline Worth, D E & 380 \\
\hline $\mathrm{Wu}, \mathrm{L}$ & 378,447 \\
\hline Wulf, S & 528 \\
\hline Wylie, A R G & 550 \\
\hline Xavier, A A P & 339 \\
\hline Xue, B & $309,310,311$ \\
\hline Yáñez-Ruiz, D R & $270,367,368,369,577$ \\
\hline Yang, C L & 398 \\
\hline Yan, $\mathrm{T}$ & $\begin{array}{l}278,299,309,310,311,321,356 \\
495,590\end{array}$ \\
\hline Yeluripati, J & 559 \\
\hline Young, E & 412 \\
\hline $\mathrm{Yu}, \mathrm{Z}$ & $415,417,418$ \\
\hline Zafarian, $\mathrm{R}$ & 330 \\
\hline Zechmeister-Boltenstern, S & 583 \\
\hline Zehetmeier, $\mathrm{M}$ & 493,528 \\
\hline Zeitz, J O & 317,458 \\
\hline Zethner, G & 583 \\
\hline Zethof, M & 404 \\
\hline Zeyer, K & 429,554 \\
\hline Zhang, T & 296 \\
\hline Zhao, G & 296,297 \\
\hline Zhao, Y G & 495 \\
\hline Zheng, W & 296 \\
\hline Zhou, X & 458 \\
\hline Zimmerman, $\mathrm{P}$ & 518 \\
\hline Zimmerman, S & 436,518 \\
\hline Zmora, $\mathrm{P}$ & 492 \\
\hline
\end{tabular}

